\title{
An investigation of Interface-GMRES(R) for fluid-structure interaction problems with flutter and divergence
}

\author{
Christian Michler • Harald van Brummelen • \\ René de Borst
}

Received: 19 December 2009 / Accepted: 7 July 2010 / Published online: 14 August 2010

(C) The Author(s) 2010. This article is published with open access at Springerlink.com

\begin{abstract}
The basic subiteration method for solving fluid-structure interaction problems consists of an iterative process in which the fluid and structure subsystems are alternatingly solved, subject to complementary partitions of the interface conditions. The main advantages of the subiteration method are its conceptual simplicity and its modularity. The method has several deficiencies, however, including a lack of robustness and efficiency. To bypass these deficiencies while retaining the main advantages of the method, we recently proposed the Interface-GMRES(R) solution method, which is based on the combination of subiteration with a NewtonKrylov approach, in which the Krylov space is restricted to the interface degrees-of-freedom. In the present work, we investigate the properties of the Interface-GMRES(R) method for two distinct fluid-structure interaction problems with parameter-dependent stability behaviour, viz., the beam problem and the string problem. The results demonstrate the efficiency and robustness of the Interface-GMRES(R) method.
\end{abstract}

Keywords Fluid-structure interaction - Subiteration . Newton-Krylov method · GMRES · Interface-GMRES . Reuse of Krylov vectors

C. Michler (凶)

Oxford University Computing Laboratory, Wolfson Building, Parks Road, Oxford OX1 3QD, UK

e-mail: Christian.Michler@comlab.ox.ac.uk

H. van Brummelen · R. de Borst

Department of Mechanical Engineering, Eindhoven University

of Technology, 5600 MB Eindhoven, The Netherlands

e-mail: E.H.v.Brummelen@ tue.nl

R. de Borst

e-mail: R.d.Borst@tue.nl

\section{Introduction}

Fluid-structure interactions (FSI) are of great relevance in aerospace, civil and offshore engineering and in biomechanics; see, e.g., Refs. [1,2,22,28-30]. Numerical methods for the aggregated fluid-structure system typically employ a partitioned solution process based on alternating solution of the fluid and structure subsystems, subject to complementary partitions of the interface conditions; see, e.g., Ref. [21]. This process, which is often referred to as subiteration, is then repeated until a preset convergence criterium is satisfied. Subiteration is a good solver for many problems, but it lacks robustness for FSI problems with large added-mass effects; cf. Refs. [7,10,31,32,35]. Moreover, in time-integration processes, subiteration is generally assigned to solve a large sequence of similar problems. Since the method has no mechanism to reuse previously generated information, it is to be considered inefficient.

To overcome these deficiencies, we proposed in [34] a novel solution method that employs subiteration as a preconditioner to GMRES; see also the proceedings article [18] for a condensed presentation of the method and [19] for an error-amplification analysis. The combination of subiteration and GMRES requires only negligible computational resources, because the GMRES acceleration can be confined to the interface degrees-of-freedom, which is considerably cheaper than applying GMRES to the aggregated equations or to the Schur complement; see, e.g., Refs. [11,16]. Thus, we refer to our method as Interface-GMRES(R), where the parenthesized $\mathrm{R}$ indicates the possibility of reusing Krylov vectors in subsequent invocations of GMRES. Such reuse can yield substantial computational savings. Since InterfaceGMRES(R) preserves the modularity of the underlying subiteration method, it can easily be implemented in codes which use subiteration as a solver. 
To place the Interface-GMRES(R) method into context, we note that investigations into efficient partitioned solution methods for fluid-structure interaction have also been presented in Refs. $[4,17]$. Alternatively, subiteration can for instance be applied as a smoother in a (space/time) multigrid procedure [35]. Further, it is to be remarked that the Interface-GMRES(R) process can in principle also be combined with subiteration methods based on a Robin-Robin partition of the interface conditions [3]. For a detailed analysis of the relation between the added mass effects and the stability and convergence of the subiteration solution method, we refer to [31]. The Interface-GMRES(R) method can also be qualified as a Schur-Newton-Krylov approach; see [5] for an advanced application of this method to aeroelastic and design-sensitivity analysis.

The Interface-GMRES method admits a re-interpretation as a Krylov-based vector extrapolation scheme; see [15] and also $[24,25]$ for earlier work on the equivalence of Krylov methods and vector extrapolation. Conversely, the Aitken extrapolation procedure (see, e.g., $[15,10]$ ) can be conceived of as an Interface-GMRES method with a onedimensional Krylov subspace. Depending on the interpretation, the methodologies can differ in certain details, such as intermediate orthonormalization of search vectors and combination with underrelaxation to gauge nonlinear effects and, accordingly, facilitate the solution of the fluid and structure subsystems. In our experience, however, orthonormalization of the search vectors is indispensable to retain a well-conditioned least squares problem in the GMRES procedure, while the combination with underrelaxation significantly reduces the cost of solving the fluid and structure subsystems. It is to be remarked that the minimal-residual property of GMRES ensures that the convergence of the Interface-GMRES method is monotonous, which is pertinent in view of the nonnormality of the subiteration process; cf. [32].

The objective of the present contribution is to examine the robustness and efficiency of the Interface-GMRES(R) method in the setting of more discriminating higher-dimensional problems than the piston problem considered in $[18,34]$. In particular, we consider the interaction of an inviscid-fluid flow with a beam and a string, respectively. The essential difference between these problems and the piston problem in the aforementioned publications is twofold. Firstly, the interface extends in both space and time, rather than only time. Consequently, the dimension of the discrete representation of the interface space is significantly higher. Secondly, the problems considered in this paper can exhibit parameter-dependent stability behaviour, e.g., stable behaviour can occur for certain parameter settings while unstable behaviour such as flutter or divergence can occur for other settings; see [9] for further elaboration of these effects.
To study the convergence behaviour of InterfaceGMRES(R) in a systematic way, we explore first the physical parameter space of the respective fluid-structure systems and we determine for which parameter settings the system is unstable, and which type of instability it exhibits. Next, we assess the convergence behaviour of Interface-GMRES(R) for representative settings of the physical and discretization parameters. We investigate the relation between the convergence behaviour of Interface-GMRES(R) and the stability of the problem. Numerical results are provided that demonstrate the performance and versatility of the Interface-GMRES(R) solution method.

This paper is organized as follows. Section 2 contains a statement of the beam and the string FSI problem. Section 3 concisely reviews the Interface-GMRES(R) solution method. In Sect. 4 we present numerical results for the beam and the string problem. Section 5 contains concluding remarks.

\section{Problem statement}

\subsection{The beam FSI problem}

Below, we present a concise description of the beam problem, for an elaboration we refer to Ref. [23]. The upper side of the beam is exposed to an airstream, and its lower side to a cavity with still air; see Fig. 1 for an illustration. The motion of the structure is described by the beam equation. Let $x, y$ and $t$ be spatial and temporal coordinates, respectively, $\alpha(x, t)$ the $y$-coordinate position of the fluid-structure interface and $L$ the length of the beam. The mathematical formulation of the fluid-structure system comprises the Euler equations on $\Omega_{\alpha}:=\{(x, y, t):-\infty<x<\infty ; \alpha(x, t)<y<\infty ; 0<$ $t<T\}$ in connection with the beam equation at the interface $\Gamma_{\alpha}:=\{(x, y, t): 0<x<L ; y=\alpha(x, t) ; 0<t<T\}$. We consider the Euler equations in conservative form:

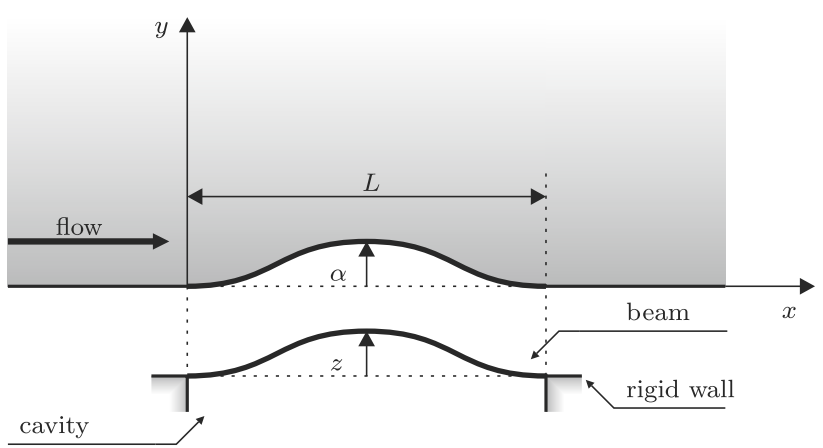

Fig. 1 Illustration of the beam FSI problem (interface region expanded for clarity) 
$\frac{\partial \mathbf{u}}{\partial t}+\frac{\partial \mathbf{f}(\mathbf{u})}{\partial x}+\frac{\partial \mathbf{g}(\mathbf{u})}{\partial y}=0, \quad(x, y, t) \in \Omega_{\alpha}$,

with

$\mathbf{u}:=\left(\begin{array}{c}\rho \\ \rho u \\ \rho v \\ E\end{array}\right), \quad \mathbf{f}(\mathbf{u}):=\left(\begin{array}{c}\rho u \\ \rho u^{2}+p(\mathbf{u}) \\ \rho u v \\ (p(\mathbf{u})+E) u\end{array}\right)$,

$\mathbf{g}(\mathbf{u}):=\left(\begin{array}{c}\rho v \\ \rho u v \\ \rho v^{2}+p(\mathbf{u}) \\ (p(\mathbf{u})+E) v\end{array}\right)$,

$p(\mathbf{u}):=(\gamma-1)\left(E-\frac{1}{2} \rho\left(u^{2}+v^{2}\right)\right)$,

and $\gamma=1.4$. In Eq. (1b), $\rho, u, v, E$ and $p$ denote the density, the $x$ - and $y$-component of the velocity, the total energy and the pressure of the fluid, respectively.

Equation (1) must be supplemented with appropriate initial and boundary conditions. On $\partial \Omega_{\alpha} \backslash \Gamma_{\alpha}$ these are prescribed by

$$
\begin{aligned}
& \mathbf{u}(x, y, 0)=\mathbf{u}^{0}(x, y), \quad-\infty<x<\infty, \\
& \alpha(x, 0)<y<\infty, \\
& (\rho v)(x, 0, t)=0, \quad x<0, x>L, \quad 0<t<T,
\end{aligned}
$$

with $\mathbf{u}^{0}(x, y)$ the given initial conditions. Condition (2b) translates into the impermeability condition of the rigid wall. Moreover, 'farfield boundary conditions' are imposed for $x \rightarrow \pm \infty$ and for $y \rightarrow \infty$. The interface conditions, i.e., the conditions on $\Gamma_{\alpha}$, are specified below.

The governing equation for the beam is:

$M \frac{\partial^{2} z}{\partial t^{2}}+D \frac{\partial^{4} z}{\partial x^{4}}=-\pi+\beta, \quad 0<x<L, 0<t<T$,

where $z$ designates the beam displacement from its equilibrium position, and the constants $M, D \in \mathbb{R}_{+}$denote the mass and the bending stiffness of the beam, respectively. The right-hand member of Eq. (3) is the forcing term which is composed of the traction $\pi$ exerted by the fluid on the structure through the interface, and the constant pressure $\beta$ in the cavity underneath the beam. The cavity pressure is equal to the freestream pressure. Eq. (3) is subject to the initial and boundary conditions

$$
\begin{aligned}
& z(x, 0)=z^{0}(x), \quad \frac{\partial z}{\partial t}(x, 0)=\dot{z}^{0}(x), \quad 0<x<L, \\
& z(0, t)=z(L, t)=0, \quad \frac{\partial z}{\partial x}(0, t)=\frac{\partial z}{\partial x}(L, t)=0, \\
& 0<t<T,
\end{aligned}
$$

with $z^{0}(x), \dot{z}^{0}(x)$ the given initial conditions. The boundary conditions (4b) state that the beam is clamped on both sides.
The Euler equations and the beam equation are connected at the interface $\Gamma_{\alpha}$ by the kinematic conditions

$$
\begin{aligned}
\left.(\rho v)\right|_{\Gamma_{\alpha}}= & \left.\rho\right|_{\Gamma_{\alpha}} \frac{\partial \alpha}{\partial t}(x, t) \\
& +\left.(\rho u)\right|_{\Gamma_{\alpha}} \frac{\partial \alpha}{\partial x}(x, t), \quad 0<x<L, \quad 0<t<T,
\end{aligned}
$$

$\alpha(x, t)=z(x, t), \quad 0<x<L, \quad 0<t<T$,

and the dynamic condition

$p\left(\left.\mathbf{u}\right|_{\Gamma_{\alpha}}\right)=\pi(x, t), \quad 0<x<L, \quad 0<t<T$.

The condition (5a) constitutes a 'slip' boundary condition, which translates into the tangency of the flow to the moving beam and renders the interface impermeable. The condition (5b) identifies the interface position and the beam position. The condition $(5 \mathrm{c})$ implies equilibrium of the forces exerted on the interface by the fluid and the structure. Note that the interface conditions are imposed on the moving boundary $\Gamma_{\alpha}$.

Upon suitable non-dimensionalization, we can identify the following dimensionless parameters that govern the behaviour of the beam fluid-structure system:

$\lambda=\frac{L C_{0}^{-1}}{M^{1 / 2} L^{2} D^{-1 / 2}}, \quad \mu=\frac{\rho_{0} L}{M}, \quad M a=\frac{V_{0}}{C_{0}}$,

where $C_{0}$ denotes the speed of sound, $\rho_{0}$ is the reference density and $V_{0}$ is the freestream velocity. The parameter $\lambda$ can be identified as the ratio of characteristic time scales of the fluid and the structure, the parameter $\mu$ constitutes the ratio of characteristic fluid mass to characteristic structure mass, and the parameter $M a$ is the Mach number.

\subsection{The string FSI problem}

Below, we present a concise description of the string FSI problem, for an elaboration we refer to Ref. [12]. The upper side of the string is exposed to an airstream, and its lower side to a cavity with still air. The problem setup corresponds to the one of the beam problem depicted in Fig. 1 with the beam replaced by a string and boundary conditions that are appropriate for a string which will be discussed below. The motion of the structure is described by the one-dimensional string equation. The mathematical formulation of the fluidstructure system comprises the Euler equations in connection with the string equation at the interface. Since the statement of the fluid subproblem and interface conditions is identical to the one for the beam problem (cf. Sect. 2.1), they will be omitted for brevity. The structure subproblem is specified below. 
The equation governing the motion of the string is

$M \frac{\partial^{2} z}{\partial t^{2}}-S \frac{\partial^{2} z}{\partial x^{2}}=-\pi+\beta, \quad 0<x<L, \quad 0<t<T$,

where $z$ is the string displacement from its equilibrium position, and the constants $M, S, L \in \mathbb{R}_{+}$denote the mass per unit length, the tension and the length of the string, respectively.

The right-hand member of Eq. (7) is the forcing term which is composed of the traction $\pi$ exerted by the fluid on the structure through the interface, and the constant pressure $\beta$ in the cavity underneath the string. The cavity pressure is equal to the freestream pressure.

Equation (7) is subject to the initial and boundary conditions

$z(x, 0)=z^{0}(x), \quad \frac{\partial z}{\partial t}(x, 0)=\dot{z}^{0}(x), \quad 0<x<L$,

$z(0, t)=z(L, t)=0, \quad 0<t<T$,

with $z^{0}(x), \dot{z}^{0}(x)$ the given initial conditions.

In contrast to the beam, the string does not provide any resistance to bending and, hence, its interaction with the fluid can be expected to be significantly different from that of the beam. Note also the different boundary conditions for the string and the beam, Eqs. (8b) and (4b), respectively.

Upon suitable non-dimensionalization, we can identify the following dimensionless parameters that govern the behaviour of the string fluid-structure system:

$\eta=\frac{L C_{0}^{-1}}{M^{1 / 2} L S^{-1 / 2}}, \quad \mu=\frac{\rho_{0} L}{M}, \quad M a=\frac{V_{0}}{C_{0}}$.

The parameter $\eta$ can be identified as the ratio of characteristic time scales of the fluid and the structure, the parameter $\mu$ constitutes the ratio of characteristic fluid mass to characteristic structure mass, and the parameter $M a$ is the Mach number. Note that the characteristic system parameters of the string and the beam problem are defined in essentially the same way; compare Eqs. (9) and (6).

A characteristic property of the string and the beam FSI problem is their ability to exhibit parameter-dependent stability behaviour. That is, the fluid-structure system can display instabilities such as flutter and divergence for certain parameter settings, whereas other parameter settings yield stable behaviour; cf. Ref. [9]. Instability of the fluid-structure system is a property that is shared by many FSI problems and that is of significant practical importance. Since flutter and divergence can induce the failure of the structure, the analysis and prediction of such instabilities play a crucial role in engineering design. For instance, in aerospace engineering, flutter and divergence impose constraints on the allowable operating conditions of aircraft. Hence, they need to be controlled by an adequate design; see, e.g., Ref. [8].

\section{The Interface-GMRES(R) solution method}

For self-containedness, we review in this section the Interface-GMRES(R) method that was recently proposed in [34] and analysed in [19]. Since the Interface-GMRES(R) method builds on the customary subiteration method, we first recall the subiteration method.

\subsection{The subiteration method}

The interconnection between the state variables and their domain of definition complicates the numerical treatment of fluid-structure interaction problems. This complication can be bypassed through an iterative solution procedure often referred to as subiteration: Given an initial approximation $z_{0}(x, t)$, for $j=1,2, \ldots$ repeat until convergence

(S1) Solve the kinematic condition: find $\alpha_{j}$ such that $\alpha_{j}(x, t)=z_{j-1}(x, t)$.

(S2) Solve the fluid on $\Omega_{\alpha_{j}}$ subject to $u_{3}\left(x, \alpha_{j}, t\right)=$ $u_{1}\left(x, \alpha_{j}, t\right) \frac{\partial \alpha_{j}}{\partial t}(x, t)+u_{2}\left(x, \alpha_{j}, t\right) \frac{\partial \alpha_{j}}{\partial x}(x, t)$ on $\Gamma_{\alpha_{j}}$ to obtain $\mathbf{u}_{j}$.

(S3) Solve the dynamic condition: find $\pi_{j}$ such that $\pi_{j}(x, t)=p\left(\mathbf{u}_{j}\left(x, \alpha_{j}(x, t), t\right)\right)$.

(S4) Solve the structure problem with right member $-\pi_{j}(x, t)+\beta$ to obtain $z_{j}(x, t)$.

This procedure obviates the simultaneous treatment of fluid and structure. Each iteration in the subiteration procedure can be conceived of as a mapping $z_{j} \mapsto z_{j+1}=\mathscr{C} z_{j}$. Accordingly, subiteration essentially constitutes a fixed-point iteration method. Momentarily assuming $\mathscr{C}$ to be linear in order to facilitate the exposition, the subiteration process is formally stable if the spectral radius of $\mathscr{C}$ is smaller than unity. However, despite formal stability, transient divergence can occur for large fluid-to-structure mass ratios or large time steps. This non-monotonous convergence behaviour is caused by nonnormality of the operator (cf. [32]) and it can lead to failure of the iterative method even if the spectral radius of the operator is less than 1. Such transient divergence compromises the robustness of the subiteration method.

\subsection{The Interface-GMRES(R) method}

The Interface-GMRES(R) method essentially constitutes a (Jacobian-free) Newton-Krylov method [6,14] applied to the interface degrees-of-freedom. To cast the nonlinear fixedpoint problem in a form that is suitable for Newton-Krylov methods, we reformulate it as $\mathscr{R} z=0$ with $\mathscr{R}:=\mathscr{C}-\mathscr{I}$ the residual operator. Correspondingly, the residual of an iterate $z_{i}$ is $r_{i}:=\mathscr{R} z_{i}=(\mathscr{C}-\mathscr{I}) z_{i}=z_{i+1}-z_{i}$. For a given initial guess $z_{0}$, Newton's method generates a sequence of 
interval to the next. Such reuse can substantially increase the efficiency of the method; however, it comes at the expense of robustness and therefore has to be exercised with some caution. We refer to the Interface-GMRES method with reuse as Interface-GMRESR. Finally, let us remark that the InterfaceGMRES(R) solution method is generic and that it is easily implemented in existing codes which use subiteration as a solver.

\section{Numerical experiments}

To demonstrate the versatility of the Interface-GMRES(R) method, we assess its convergence behaviour on the beam and the string FSI problem in Sects. 4.2 and 4.4, respectively. In particular, we investigate the effect of physical instability due to flutter and divergence on Interface-GMRES(R) convergence and on the effectiveness of reuse of the Krylov space. For reference purposes, we include comparisons with standard subiteration.

\subsection{Experimental setup of the beam FSI problem}

We consider the beam problem stated in Sect. 2.1. The infinite-dimensional domain with $x \rightarrow \pm \infty$ and $y \rightarrow \infty$ is truncated. In the $x$-direction inflow and outflow fluid boundary conditions are prescribed with the flow going from left to the right, and in the $y$-direction the domain is bounded by a solid wall at a distance of one from the beam. The distance of the solid wall to the beam is sufficiently large to ensure that the wall does not significantly influence the solution and the convergence behaviour of the solution methods.

We use initial conditions for the beam according to its first mode shape. The initial conditions for the fluid are determined as the steady-state solution of the flow over a beam that is deflected according to its first mode shape. The system parameters are given in Table 1, where $\tau$ denotes the length of the solution time interval. With $M a=1.5$, the flow is supersonic.

The fluid-structure system is discretized by the space/time finite-element method with piecewise-polynomial basis functions that are discontinuous in time and continuous in space; see also Refs. [26,27] for the space/time finite-element method for problems with moving boundaries. As basis functions for the structure discretization we use Legendre

Table 1 System parameters for the beam FSI problem (* indicates a variable parameter)

\begin{tabular}{lllll}
\hline Case & $\lambda$ & $\mu$ & $M a$ & $\tau$ \\
\hline I & 0.25 & $*$ & 1.5 & 0.05 \\
II & $*$ & 10 & 1.5 & 0.05 \\
\hline
\end{tabular}

polynomials, and enforce $C^{1}$-continuity in space by means of Lagrange multipliers. The basis functions for the fluid are of modal type in conformity with Ref. [13, ch.3].

The time-discontinuous Galerkin discretization implies that displacement and velocity of the structure are discontinuous from one time slab to the next. However, since the fluidboundary representation assumes a continuous displacement, the discontinuity in the structure displacement needs to be controlled. To render the discontinuity in the structure displacement and velocity negligible, we use polynomials of sufficiently high order for the approximation of the structure. It is to be remarked that the considered discretization does not maintain the conservation properties at the fluidstructure interface; cf. Refs. [20,33]. To render the error pertaining to the lack of conservation negligible, we choose a discretization for fluid and structure that is sufficiently fine.

The discretization parameters are given in Table 2, where the polynomial degree of the approximation spaces associated with $\mathbf{u}, \alpha, z$ and $\pi$ are, respectively, $\left(P_{\mathbb{U}}^{x}, P_{\mathbb{U}}^{y}, P_{\mathbb{U}}^{t}\right)$, $\left(P_{\mathbb{A}}^{x}, P_{\mathbb{A}}^{t}\right),\left(P_{\mathbb{Z}}^{x}, P_{\mathbb{Z}}^{t}\right)$ and $\left(P_{\mathbb{P}}^{x}, P_{\mathbb{P}}^{t}\right)$, and the number of elements, $N$, is denoted accordingly. The number of elements in the $x$-direction is specified over the length of the beam. The discretization time step is equal to the length of the solution time interval. The discretization is sufficiently fine to ensure that the results are essentially mesh independent.

In each time slab, we provide an initial approximation of the structure displacement based on a linear extrapolation of the initial conditions according to

$z_{0}(x, t)=z^{0}(x)+\dot{z}^{0}(x) t, \quad 0 \leq x \leq L, \quad 0 \leq t \leq \tau$.

We set the convergence tolerance to $\epsilon_{0}=10^{-4}\left\|r_{0}\right\|$, i.e., we require a reduction of the initial residual by four orders of magnitude. In addition, we specify for the Newton-Krylov method the tolerance for the GMRES iteration according to $\epsilon_{1}=10^{-1}\left\|r_{i}\right\|$, i.e., we use a relative tolerance for the convergence in the inner loop of the acceleration; cf. Sect. 3.2. Moreover, the underrelaxation parameter is set to $v=10^{-2}\left\|r_{0}\right\|$ for the Interface-GMRES method with reuse and to $v=10^{-2}\left\|r_{i}\right\|$ for the method without reuse.

\subsection{Numerical results for the beam FSI problem}

In the first test case, we study the convergence of the Interface-GMRES(R) method and subiteration for three distinct

Table 2 Discretization parameters for the beam and the string FSI problems

\begin{tabular}{llllllll}
\hline$N_{\mathbb{U}}$ & $N_{\mathbb{A}}$ & $N_{\mathbb{Z}}$ & $N_{\mathbb{P}}$ & $P_{\mathbb{U}}$ & $P_{\mathbb{A}}$ & $P_{\mathbb{Z}}$ & $P_{\mathbb{P}}$ \\
\hline$(16,24,1)$ & $(16,1)$ & $(16,1)$ & $(16,1)$ & $(2,2,2)$ & $(1,1)$ & $(6,6)$ & $(2,2)$
\end{tabular}


Fig. 2 Beam FSI problem, test case I: space/time displacement of the beam (colour bars) for system parameters according to Table 1 and $\mu=1$ (left),$\mu=50$ (center) and $\mu=100$ (right)
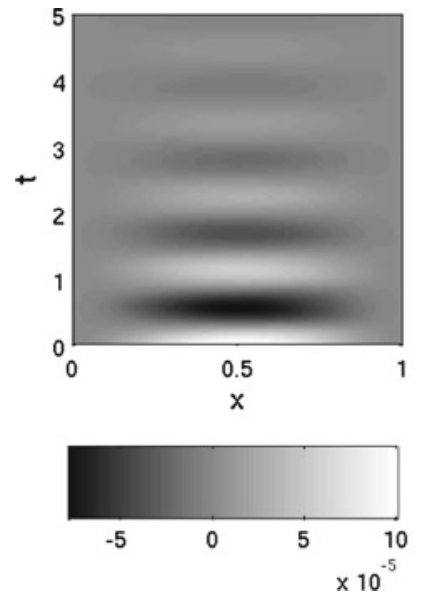
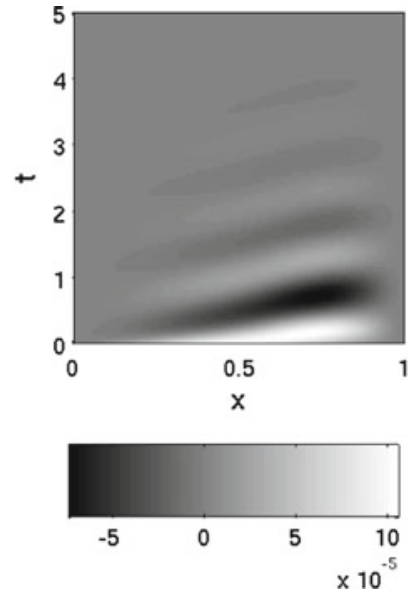
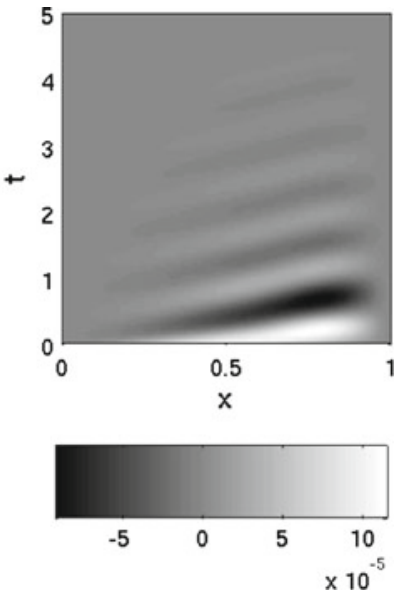

settings of the problem with parameters as given in Table 1, case $\mathrm{I}$ and $\mu=1,50,100$. We remark that the added mass and the spectral radius of the subiteration-operator derivative scale with $\mu$; see also Ref. [32].

Figure 2 plots the displacement of the beam in space/time. For all considered settings, the oscillation of the structure attenuates with time, indicating that the fluid-structure system is stable. Moreover, it is apparent that the beam deflection is downwind according to the direction of the flow. The convergence behaviour of the Newton-Krylov method with and without reuse and of the subiteration method is displayed in Fig. 3 for time steps 1 and 50 for exemplification. In addition, we plot in Figs. 4 and 5 the dimension of the Krylov space and the cumulative number of iterations versus the time-step counter, respectively. The cumulative number of iterations specifies the total number of iterations required for convergence up to and including the time step under consideration. Figure 3 illustrates that if reuse is applied, initially most iterations of the Newton-Krylov method are spent on generating the Krylov space. However, in subsequent time steps, increasingly fewer Krylov vectors need to be added to the space due to reuse; see also Fig. 4. This results in a decreasing number of iterations per time step and manifests in the gradually changing slope of the cumulative-iteration-count curve; see Fig. 5. In contrast, the number of iterations required by subiteration hardly changes in subsequent time steps. We infer from these results that reuse can render the NewtonKrylov method computationally cheaper than subiteration even under conditions that are favorable for the convergence of subiteration; see Figs. 3 (left) and 5 (left) with $\mu=1$. Subiteration convergence deteriorates significantly with increasing $\mu$, in contrast to Newton-Krylov convergence. Hence, a discrepancy in computational cost for larger $\mu$ emanates. For $\mu=100$, subiteration diverges. Note that the NewtonKrylov method attains convergence despite the instability of the underlying subiteration method.

For reference, we have included in Figs. 3 and 5 the results for the Newton-Krylov method without reuse of the Krylov space. A comparison to the method with reuse clearly demonstrates the significant savings in computational cost that can be obtained by reusing the Krylov space.

To put our results into context, we remark that for an initial amplitude of the beam deflection of approximately $10^{-4}$ the system behaviour is close to linear. Preliminary studies indicate that for nonlinear system behaviour corresponding to larger initial amplitudes the performance of the Newton-Krylov method degrades only moderately. Moreover, we remark that our results are in good agreement with the results obtained on the piston model problem; cf. [18,34].

In the second test case, we investigate the effect of physical instability on convergence and on the effectiveness of reusing the Krylov space. To this end, we consider the fluid-structure system with parameters according to Table 1 , case II and two representative settings of $\lambda$, viz., $\lambda=0.1$ and $\lambda=0.25$. The discretization parameters are specified in Table 2.

Figure 6 plots the numerical solution of the beam displacement in space/time for the unstable system (left figure) and the stable system (right). Whereas for $\lambda=0.1$ the oscillation amplifies which indicates flutter, for $\lambda=0.25$ the oscillation attenuates, indicating stability of the fluidstructure system. Figure 7 (left) plots the cumulative number of iterations versus the time-step counter for the Newton-Krylov method and for subiteration as a reference. In addition, Fig. 7 (right) plots the dimension of the Krylov space versus the time-step counter. We remark that these figures plot up to a time step of $n=200$ corresponding to computational time $t=10$, whereas Fig. 6 plots only up to $n=100(t=5)$. Note that the instability becomes increasingly pronounced with time. Figure 7 (left) displays a slight change in slope of the cumulative-iteration-count curve of the Newton-Krylov method with reuse for the unstable system setting. To explain this change in slope, we consider the evolution of the Krylov-space dimension plotted in Fig. 7 (right). The figure exhibits that, after the initial construction of a sufficiently large Krylov space, the dimension of the space remains essentially constant up to a time step of 
Fig. 3 Beam FSI problem, test case I: residual reduction in the $L^{2}$ norm versus iteration number in time steps 1 (top) and 50 (bottom) for the Newton-Krylov method with reuse (continuous line) and without reuse (dashed line) and for subiteration (dotted line); residual estimates and true residuals of the Newton-Krylov method are indicated by open circle and open square, respectively, and residuals of subiteration by open triangle; $\mu=1$ (left),$\mu=50$ (center) and $\mu=100$ (right). $y$ axis in $\log _{10}$-scale
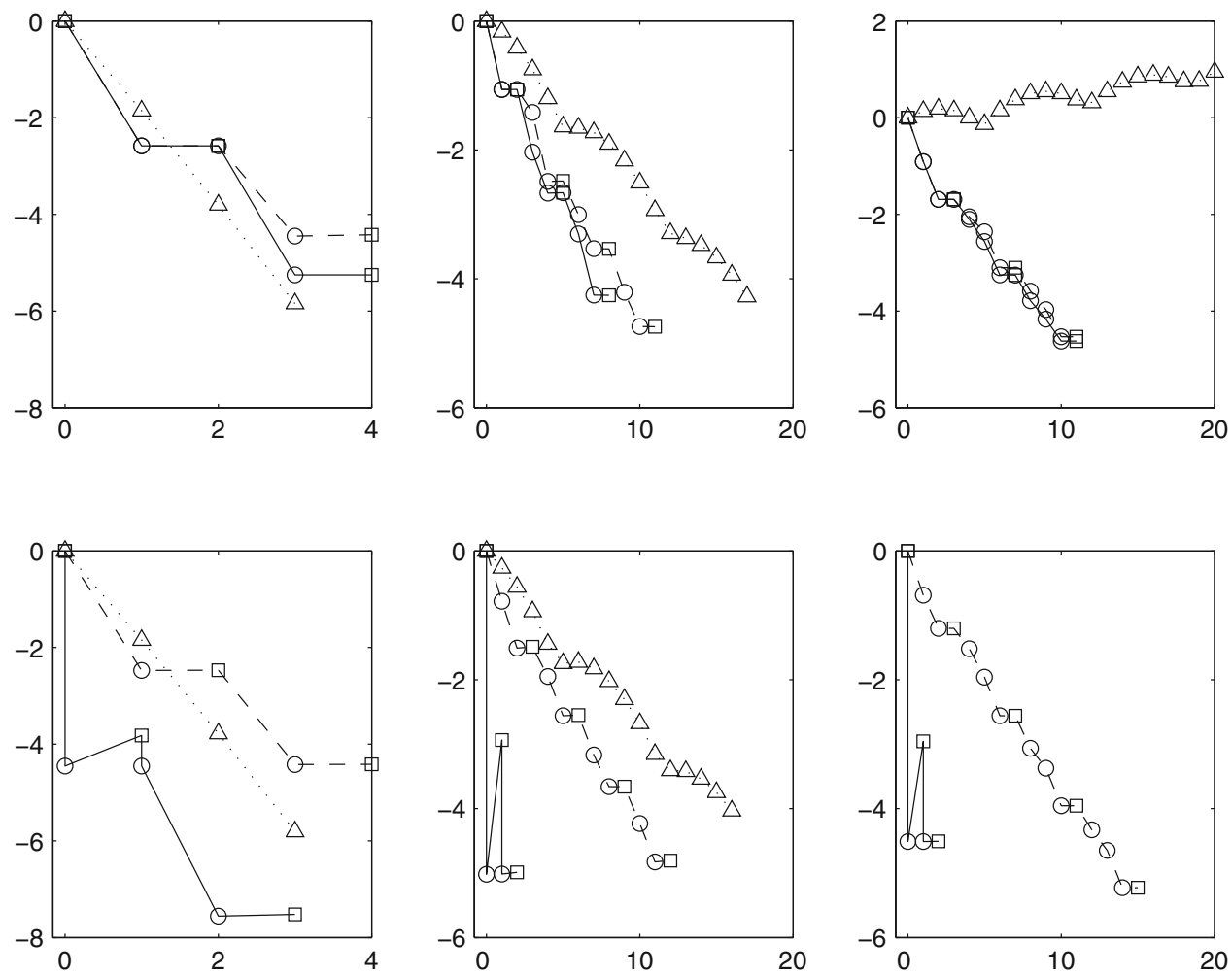

Fig. 4 Beam FSI problem, test case I: dimension of the Krylov space versus the time-step counter for the Newton-Krylov method with reuse in subsequent time steps; $\mu=1$ (left), $\mu=50$ (center) and $\mu=100$ (right)
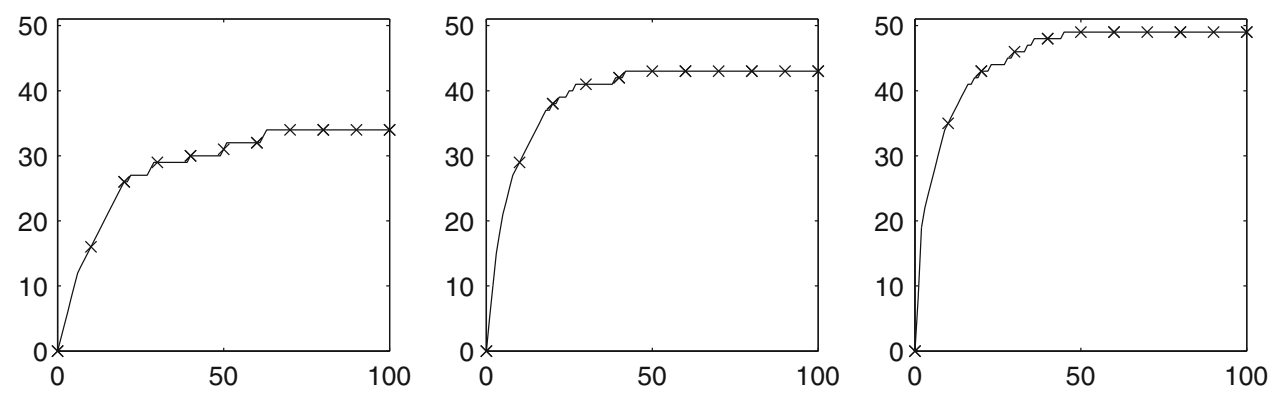

Fig. 5 Beam FSI problem, test case I: cumulative number of iterations versus the time-step counter for the Newton-Krylov method with reuse (continuous line) and without reuse (dashed line) and for subiteration (dotted line); $\mu=1$ (left), $\mu=50$ (center) and $\mu=100$ (right)
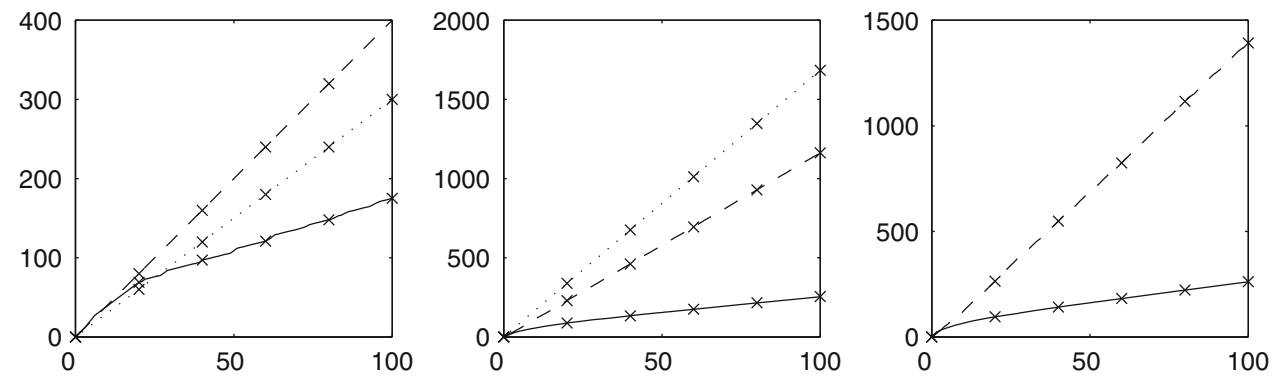

approximately 100 . Henceforth, the dimension of the Krylov space further increases in the case of the unstable system, which means that additional Krylov vectors need to be added to the space to attain convergence. This indicates a mild degradation in the effectiveness of the reused Krylov space which can be attributed to the significant change in the solution induced by flutter. However, this effect appears to be minor in that reuse remains beneficial and renders Newton-Krylov convergence faster than subiteration convergence; see Fig. 7 (left).

Summarizing, the test cases show that the InterfaceGMRES method is much more efficient than subiteration separately. Settings corresponding to a relatively weak coupling in the FSI problem, e.g. due to small $\mu$, are favorable for 
Fig. 6 Beam FSI problem, test case II: space/time displacement of the beam (colour

bars)—solution computed with system parameters according to Table 1 with $\lambda=0.1$ (left) and $\lambda=0.25($ right $)$
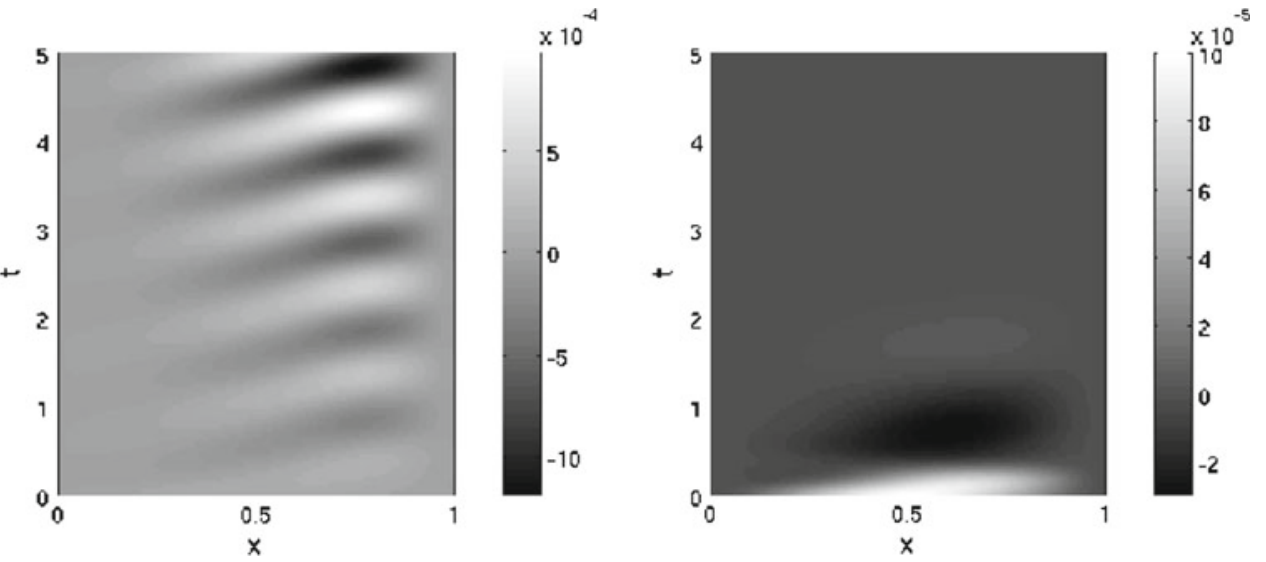

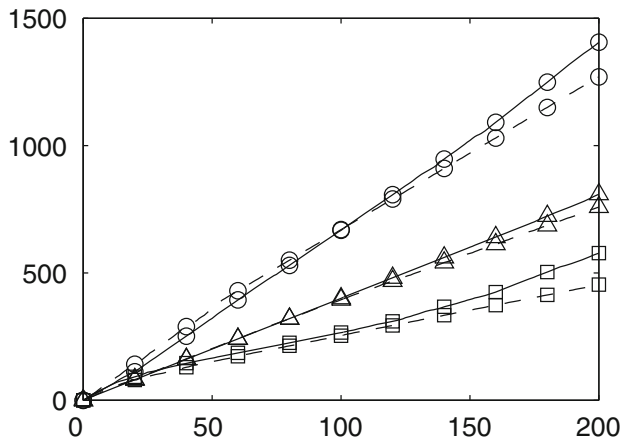

Fig. 7 Beam FSI problem, test case II: cumulative number of iterations versus the time-step counter for the Newton-Krylov method with reuse in subsequent time steps (open square) and without reuse (open circle) and for subiteration (open triangle) (left), and dimension of the Krylov

the subiteration method. For such settings, the convergence behaviour of subiteration and Interface-GMRES is comparable. For larger $\mu$ and, accordingly, a stronger coupling, Interface-GMRES converges much faster than subiteration. Even if the coupling is so strong that the subiteration method separately diverges, the Interface-GMRES method still displays adequate convergence behaviour. Moreover, if the reuse option is exercised, then the Interface-GMRESR method converges in just a few iterations, independent of the strength of the coupling.

\subsection{Experimental setup of the string FSI problem}

To further validate the Interface-GMRES method, we consider the string problem from Sect. 2.2. The infinitedimensional domain is truncated in the same manner as for the beam FSI problem; cf. Sect. 4.1.

We use initial conditions for the string corresponding to its first mode shape and an amplitude of $10^{-4}$. The initial conditions for the fluid are determined as the steady-state solution of the flow for this initial configuration. It is to be noted that, in contrast to the beam problem, for the string problem

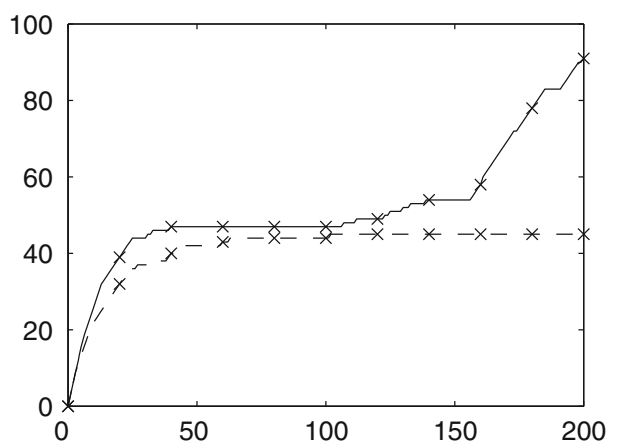

space versus the time-step counter for the Newton-Krylov method with reuse (right); system parameters according to Table 1 with $\lambda=0.1$ (continuous line) and $\lambda=0.25$ (dashed line)

Table 3 System parameters for the string FSI problem

\begin{tabular}{lllll}
\hline Case & $\eta$ & $\mu$ & $M a$ & $\tau$ \\
\hline I & 1.0 & 0.01 & 1.2 & 0.05 \\
II & 1.0 & 1.0 & 0.5 & 0.05 \\
III & 0.53 & 1.0 & 1.1 & 0.05 \\
\hline
\end{tabular}

we shall apply a geometric linearization. This implies that the fluid domain remains undeformed. The fluid velocity at the interface, however, is determined by the velocity of the structure in accordance with the kinematic interface condition (5a). The reason for this geometric linearization is that we employ a discontinuous Galerkin method for the string, which would otherwise impose a discontinuous deformation of the fluid domain. Let us remark, however, that the effect of such a geometric linearization is in general small.

The system parameters for three representative types of behaviour of the string fluid-structure system are given in Table 3. We remark that these settings are conservative in the sense that they are favorable for the subiteration method on account of the small fluid-to-structure mass ratio $\mu$. 
The fluid-structure system is discretized by the space/time finite-element method with piecewise-polynomial basis functions. The discretization method for the fluid equations is identical to the one used for the beam problem; cf. Sect. 4.1. For the discretization of the one-dimensional string we use a space/time discontinuous Galerkin method. This choice is motivated by the fact that the string is represented by the second-order wave equation (7), which is of hyperbolic type. To obtain the discontinuous Galerkin formulation, we recast Eq. (7) into a first-order hyperbolic system in terms of $u_{s}=\partial z / \partial t$ and $v_{s}=\partial z / \partial x$. The displacement of the string $z$ can then be obtained by integration of $u_{s}$. The aggregated system for $\left(u_{s}, v_{s}, z\right)$ is discretized in space/time by means of the discontinuous Galerkin method with Legendre polynomials as basis functions. Neighbouring elements are connected

Fig. 8 String FSI problem: space/time displacement of the string; test case I exhibiting a stable solution (left), case II exhibiting divergence (center) and case III exhibiting flutter (right)
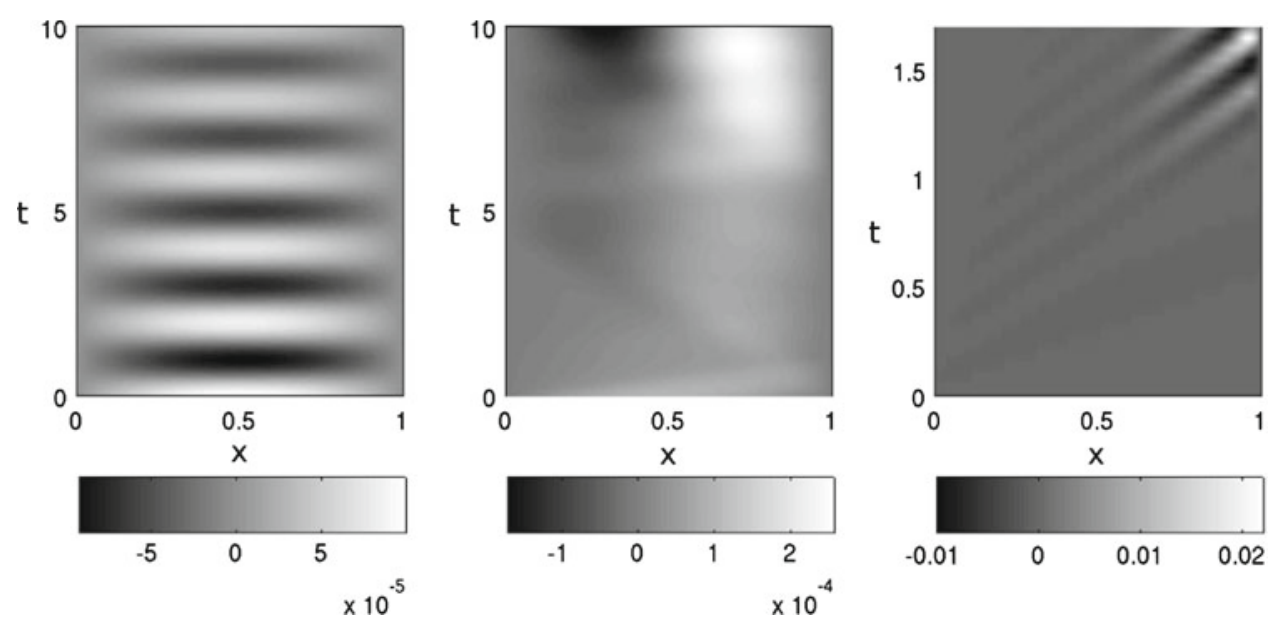

Fig. 9 String FSI problem, test cases I-III: residual reduction in the $L^{2}$ norm versus iteration number for the Newton-Krylov method with reuse (continuous line) and without reuse (dashed line) and for subiteration (dotted line); residual estimates and true residuals of the Newton-Krylov method are indicated by open circle and open square, respectively, and residuals of subiteration by open triangle; time steps 1 (top) and 50 (bottom) for test cases I (left) and II (center), and time steps 1 (top) and 30 (bottom) for case III (right)
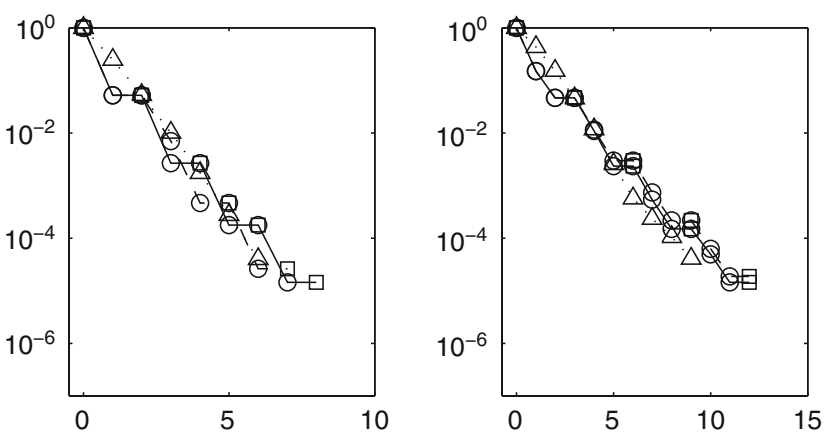

by inter-element fluxes that are determined from the solution of the Riemann problem on inter-element boundaries. For details of the structure discretization we refer to Ref. [12]. The discretization parameters for the string problem for test cases I-III are taken to be identical to the ones for the beam problem given in Table 2.

To provide an initial approximation for the iterative solution procedure, in each time slab, the structure displacement is initialized with the solution of the previous time slab. We set the convergence tolerance to $\epsilon_{0}=10^{-4}\left\|r_{0}\right\|$. In addition, we specify for the Newton-Krylov method the tolerance for the GMRES iteration according to $\epsilon_{1}=10^{-1}\left\|r_{i}\right\|$. Moreover, the underrelaxation parameter is set to $v=\left\|r_{0}\right\|$ for the Newton-Krylov method with reuse and to $v=\left\|r_{i}\right\|$ for the method without reuse.
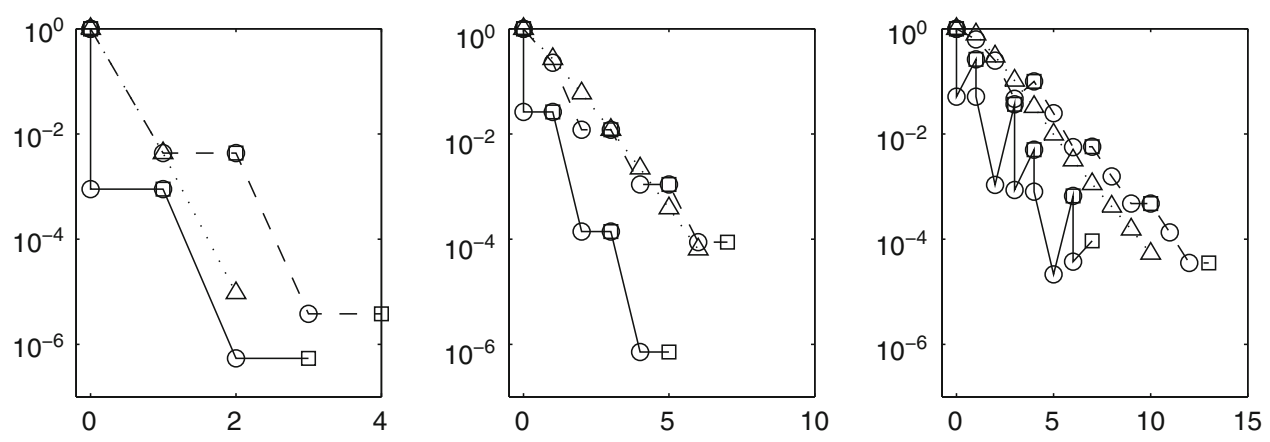
Fig. 10 String FSI problem, test cases I-III: dimension of the Krylov space versus the time-step counter for the Newton-Krylov method with reuse in subsequent time steps; test case I (left), case II (center) and case III (right)

Fig. 11 String FSI problem, test cases I-III: cumulative number of iterations versus the time-step counter for the Newton-Krylov method with reuse (continuous line) and without reuse (dashed line) and for subiteration (dotted line); test case I (left), case II (center) and case III (right)
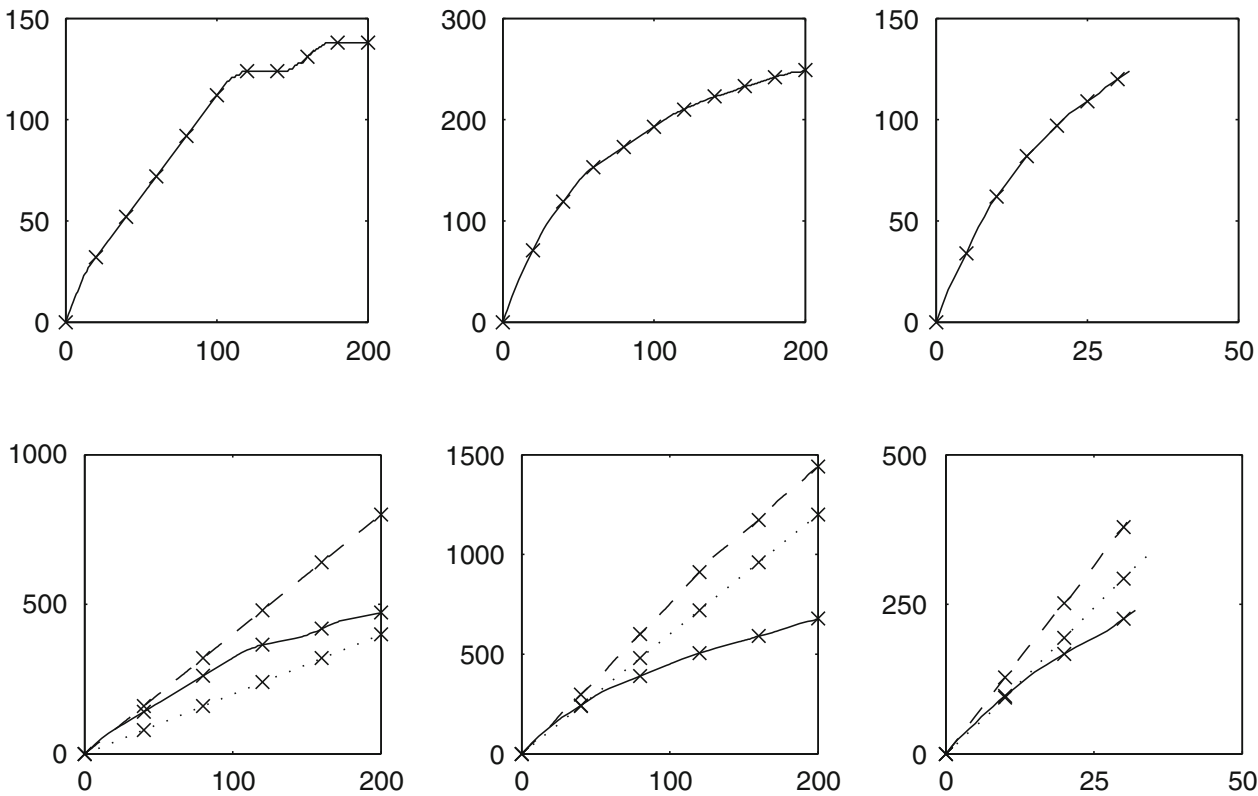

\subsection{Numerical results for the string FSI problem}

We consider three parameter settings for the string problem; see Table 3. The displacement of the string in space/time is displayed in Fig. 8 for the three cases under consideration. Whereas Fig. 8 (left) shows a displacement that decays with time, indicating stability of the fluid-structure system, Fig. 8 (center and right) displays a solution whose amplitude grows with time, indicating instability of the fluid-structure system. Figure 8 (center) displays a diverging solution. Let us remark that for a specific range of subsonic Mach numbers, the occurrence of divergence can be explained by Bernoulli's theorem, as the pressure force can be larger than the retraction force by the longitudinal tension in the string. Figure 8 (right) shows an oscillatory solution with growing amplitude, indicating flutter. In anticipation of subsequent plots, we remark that for the flutter case the computation fails in time step 34 $(t=1.7)$ in the solution of the fluid subsystem. This failure can be attributed to the amplifying oscillatory flutter solution that induces large velocities at the fluid boundary, which eventually leads to failure of the fluid solver.

The convergence behaviour of the Newton-Krylov method with and without reuse and of subiteration is displayed in Fig. 9 for time steps 1 and 50 for test cases I and II and for time steps 1 and 30 for test case III for exemplification. In addition, we plot in Figs. 10 and 11, respectively, the dimension of the Krylov space for the Newton-Krylov method with reuse and the cumulative number of iterations versus the time-step counter.

Figures 9 and 11 demonstrate that the improvement in efficiency that can be gained by reuse is not restricted to stable fluid-structure systems only but also applies to systems undergoing divergence or flutter. In the case of flutter, the
Newton-Krylov method with reuse exhibits 'jumps' between the residual estimate and the actual nonlinear residual; see Fig. 9 (bottom right). This disparity between estimate and actual residual indicates a mild degradation in the effectiveness of the reused Krylov space that can be attributed to the significant change in the solution induced by flutter. Nevertheless, reuse remains beneficial and renders Newton-Krylov convergence faster than subiteration convergence; see Figs. 9 (bottom right) and 11 (right). This observation is consistent with the results obtained for the beam problem. In the case of divergence, and at variance with flutter, the Newton-Krylov method with reuse does not exhibit a significant discrepancy between the residual estimates and the true residuals; see Fig. 9 (center). Moreover, Fig. 11 shows that the reduction in cost obtained by reusing the Krylov space is more pronounced in the case of divergence than for flutter. This indicates that a monotonically diverging solution is more amenable to reuse than an oscillatory flutter solution. Presumably this is connected with the number of modes that participate in the unstable behaviour, as all such modes must eventually be incorporated in the Krylov space.

Figure 11 shows that for the considered cases subiteration is computationally cheaper than the Newton-Krylov method without reuse. However, with reuse, the NewtonKrylov method is computationally more efficient than subiteration. We remark that for a system behaviour that is close to linear on account of the small initial string-deflection amplitude of $10^{-4}$, savings in the number of Newton iterations are possible by using a more stringent value for the relative tolerance $\epsilon_{1}$. Moreover, let us emphasize that the chosen system parameters are favorable for subiteration, on account of the small mass ratio and small time step. For larger mass ratios and time steps, the convergence behaviour of the sub- 
iteration method deteriorates and the effectiveness of the Newton-Krylov method becomes more pronounced; see Ref. [34] and also Sect. 4.2.

A comparison of the results for the beam and the string FSI problem conveys that the required dimension for the Krylov space is in general higher for the string problem than for the beam problem; compare Figs. 10 and 4. This phenomenon can be explained by the fact that the eigenvalues of the structural operator increase more rapidly for the beam than for the string. Accordingly, to achieve a certain prescribed residual reduction, the Krylov space for the beam needs to contain fewer vectors than for the string. Nevertheless, the Interface-GMRESR method provides adequate convergence behaviour also for the string problem.

\section{Conclusions}

In this paper we assessed the convergence behaviour of the Interface-GMRES(R) solution method for FSI problems on two prototypical model problems, viz., the beam and the string FSI problem. These model problems exhibit parameter-dependent stability behaviour, admitting instabilities such as flutter and divergence.

Our numerical experiments demonstrate that the InterfaceGMRES method is much more robust than the subiteration method separately. Moreover, Interface-GMRES(R) generally converges faster, especially for the $(R)$ version with reuse of the Krylov space. If the fluid-structure coupling is weak, e.g. for small fluid-to-structure mass ratios, the InterfaceGMRES method without reuse can be slightly less efficient than the subiteration method, on account of the inherent linearization in the Interface-GMRES method which is absent in the subiteration method. For strongly-coupled problems, the Interface-GMRES method clearly outperforms the subiteration method. Moreover, the Interface-GMRES method even converges in cases where the underlying subiteration method diverges, e.g. for large fluid-to-structure mass ratios.

Our results indicate that physical instability in the form of flutter can induce a mild degradation of the effectiveness of reuse of the Krylov space. However, this degradation appears to be minor and we observed a beneficial effect of reuse in all considered cases. Moreover, the degradation appears to be less pronounced for divergence-type instabilities than for flutter-type instabilities.

Acknowledgments The authors would like to thank Rob in't Groen for his assistance in setting up the string FSI problem.

Open Access This article is distributed under the terms of the Creative Commons Attribution Noncommercial License which permits any noncommercial use, distribution, and reproduction in any medium, provided the original author(s) and source are credited.

\section{References}

1. Bazilevs Y, Calo VM, Zhang Y, Hughes TJR (2006) Isogeometric fluid-structure interaction analysis with applications to arterial blood flow. Comput Mech 38:310-322

2. Bazilevs Y, Hsu MC, Kiendl J, Wüchner R, Bletzinger KU (2010) 3D simulation of wind turbine rotors at full scale. Part II. Fluidstructure interaction. Int J Numer Methods Fluids (submitted)

3. Badia S, Nobile F, Vergara C (2009) Robin-Robin preconditioned Krylov methods for fluid-structure interaction problems. Comput Methods Appl Mech Eng 198(33-36):2768-2784

4. Badia S, Quaini A, Quarteroni A (2008) Modular vs. non-modular preconditioners for fluid-structure systems with large addedmass effect. Comput Methods Appl Mech Eng 197(49-50):42164232

5. Barcelos M, Bavestrello H, Maute K (2006) A Schur-NewtonKrylov solver for steady-state aeroelastic analysis and design sensitivity analysis. Comput Methods Appl Mech Eng 195(17-18): 2050-2069

6. Brown PN, Saad Y (1990) Hybrid Krylov methods for nonlinear systems of equations. SIAM J Scientific Stat Comput 11:450-481

7. Causin P, Gerbeau JF, Nobile F (2005) Added-mass effect in the design of partitioned algorithms for fluid-structure problems. Comput Methods Appl Mech Eng 194:4506-4527

8. Cunningham AM (2003) Buzz, buffet and LCO on military aircraft-the aeroelastician's nightmares. Proceedings of the International Forum on Aeroelasticity and Structural Dynamics (IFASD) (Amsterdam, The Netherlands, 4-6 June 2003), National Aerospace Laboratory (NLR), The Netherlands

9. Dowell EH (1975) Aeroelasticity of plates and shells. Monographs and textbooks on mechanics of solids and fluids. Noordhoff, Leiden

10. Förster C, Wall WA, Ramm E (2007) Artificial added mass instabilities in sequential staggered coupling of nonlinear structures and incompressible viscous flows. Comput Methods Appl Mech Eng 196:1278-1293

11. Heil M (2004) An efficient solver for the fully-coupled solution of large-displacement fluid-structure interaction problems. Comput Methods Appl Mech Eng 193:1-23

12. in't Groen R, van Brummelen EH (2006) Numerical investigation of the membrane fluid-structure interaction problem. Technical Report DACS-06-001, Delft University of Technology, The Netherlands. Available at: http://www.em.lr.tudelft.nl

13. Karniadakis GE, Sherwin SJ (1999) Spectral/hp element methods for CFD. Oxford University Press, New York

14. Knoll DA, Keyes DE (2004) Jacobian-free Newton-Krylov methods: a survey of approaches and applications. J Comput Phys 193(2):357-397

15. Küttler U, Wall WA (2009) Vector extrapolation for strong coupling fluid-structure interaction solvers. J Appl Mech 76(2). doi:10. $1115 / 1.3057468$

16. Matthies HG, Steindorf J (2003) Partitioned strong coupling algorithms for fluid-structure interaction. Comput Struct 81:805812

17. Michler C (2005) Efficient numerical methods for fluid-structure interaction. Dissertation, Delft University of Technology, The Netherlands. Available from: http://repository.tudelft.nl/

18. Michler C, van Brummelen EH, de Borst R (2005) An interface Newton-Krylov solver for fluid-structure interaction. Int J Numer Methods Fluids 47:1189-1195. Special issue containing the Proceedings of the Eighth ICFD Conference on Numerical Methods for Fluid Dynamics (Oxford, UK, March 29-April 1, 2004)

19. Michler C, van Brummelen EH, de Borst R (2006) Erroramplification analysis of subiteration-preconditioned GMRES for fluid-structure interaction. Comput Methods Appl Mech Eng 195: 2124-2148 
20. Michler C, van Brummelen H, Hulshoff S, de Borst R (2003) The relevance of conservation for stability and accuracy of numerical methods for fluid-structure interaction. Comput Methods Appl Mech Eng 192:4195-4215

21. Morton SA, Melville RB, Visbal MR (1997) Accuracy and coupling issues of aero-elastic Navier-Stokes solutions on deforming meshes. AIAA 1085:252-262

22. Nordsletten D, Kay D, Smith N (2010) A non-conforming monolithic finite element method for problems of coupled mechanics. J Comput Phys (under review)

23. Piperno S, Farhat C (2001) Partitioned procedures for the transient solution of coupled aeroelastic problems. Part II. Energy transfer analysis and three-dimensional applications. Comput Methods Appl Mech Eng 190:3147-3170

24. Sidi A (1988) Extrapolation vs. projection methods for linear systems of equations. J Comput Appl Math 22:71-88

25. Sidi A (1991) Efficient implementation of minimal polynomial and reduced rank extrapolation methods. J Comput Appl Math 36: 305-337

26. Tezduyar TE, Behr M, Liou J (1992) A new strategy for finite element computations involving moving boundaries and interfacesthe deforming-spatial-domain/space-time procedure. I. The concept and the preliminary numerical tests. Comput Methods Appl Mech Eng 94:339-351

27. Tezduyar TE, Behr M, Mittal S, Liou J (1992) A new strategy for finite element computations involving moving boundaries and interfaces - the deforming-spatial-domain/space-time procedure: II. Computation of free-surface flows, two-liquid flows, and flows with drifting cylinders. Comput Methods Appl Mech Eng 94: $353-371$
28. Tezduyar T, Sathe S, Cragin T, Nanna B, Conklin B, Pausewag J, Schwaab M (2007) Modelling of fluid-structure interactions with the space-time finite elements: arterial fluid mechanics. Int J Numer Methods Fluids 54:901-922

29. Tezduyar TE, Sathe S, Pausewang J, Schwaab M, Christopher J, Crabtree J (2008) Fluid-structure interaction modeling of ringsail parachutes. Comput Mech 43:133-142

30. Torii R, Oshima M, Kobayashi T, Takagi K, Tezduyar TE (2009) Fluid-structure interaction modeling of blood flow and cerebral aneurysm: significance of artery and aneurysm shapes. Comput Methods Appl Mech Eng 198:3613-3621

31. van Brummelen EH (2009) Added mass effects of compressible and incompressible flows in fluid-structure interaction. J Appl Mech 76(2). doi:10.1115/1.3059565

32. van Brummelen EH, de Borst R (2005) On the nonnormality of subiteration for a fluid-structure-interaction problem. SIAM J Scientific Comput 27:599-621

33. van Brummelen EH, Hulshoff SJ, de Borst R (2003) Energy conservation under incompatibility for fluid-structure interaction problems. Comput Methods Appl Mech Eng 192:2727-2748

34. van Brummelen EH, Michler C, de Borst R (2005) InterfaceGMRES(R) acceleration of subiteration for fluid-structure-interaction problems. Technical Report DACS-05-001, Delft University of Technology, The Netherlands. Available at: http://www.em.lr. tudelft.nl

35. van Brummelen EH, van der Zee KG, de Borst R (2008) Space/ time multigrid for a fluid-structure-interaction problem. Appl Numer Math 58:1951-1971 